\title{
DAS RECHT ZUR INTERAFRIKANISCHEN INTERVENTION
}

\author{
Von Dieter Schröder
}

\section{Das Recht zur Intervention im allgemeinen Völkerrecht}

Trotz des heute allgemein anerkannnten Verbots der Intervention ${ }^{1}$ zeigt sich weltweit eine wachsende Neigung zur Intervention, die u. a. auch in Afrika zu beobachten ist. Diese Beobachtung kontrastiert auf den ersten Blick mit der „Declaration on the Inadmissibility of Intervention in the Domestic Affairs of States and the Protection of their Independence and Sovereignty", die die Vollversammlung der Vereinten Nationen auf Betreiben der Sowjetunion und der Tschechoslowakei mit Unterstützung der afrikanischen Staaten im Jahre 1966 angenommen hat ${ }^{2}$. In dieser Deklaration heißt es:

"1. No State has the right to intervene, directly or indirectly, for any reason whatever, in the internal or external affairs of any other State. Consequently, armed intervention and all other forms of interference or attempted threats against the personality of the State or against the political, economic and cultural elements, are condemned.

5. Every State has an inalienable right to choose its political, economic, social and cultural systems, without interference in any form by another State."

Hier ist allerdings nur von Maßnahmen einzelner Staaten gegen einzelne Staaten die Rede, nicht jedoch von kollektiven Interventionen, wie sie beispielsweise unter der Breschnew-Doktrin ${ }^{3}$ im Herbst 1968 gegen die Tschechoslowakei von Mitgliedern des Warschauer Paktes vollzogen wurde. Die Deklaration hat allerdings auch den Fall der Kollektiv-Intervention bedacht. Dazu heißt es:

"7. For the purpose of the present declaration, the term 'State' covers both individual States and groups of States."

Trotz des umfassenden Bekenntnisses zum Interventionsverbot enthält die Deklaration eine Klausel, die die Zulässigkeit von rassisch oder antikolonialistisch begründeter Intervention implizieren könnte:

"6. All States shall respect the right of self-determination and independence of peoples and nations, to be freely exercised without any foreign pressure, and with absolute respect to human rights and fundamental freedom. Consequently, all States shall contribute to the complete elimination of racial discrimination and colonialism in all its forms and manifestations."

Demnach stehen Maßnahmen zur völligen Beseitigung von Rassendiskriminierung und "Kolonialismus" nicht im Widerspruch zum Interventionsverbot. Dies ist zwar nur eine durch die Vollversammlung der Vereinten Nationen festgestellte Rechtsüberzeugung aller Mitgliedstaaten und keineswegs ein Völkerrechtssatz. Diese Rechtsüberzeugung ist aber durchaus geeignet, zur Ausbildung neuen Völkergewohnheitsrechts beizutragen. Bereits wenige Tage nach dem Beschluß über die Deklaration hat auch schon die Vollversammlung den Staaten empfohlen, eine

1 Vgl. dazu Axel Gerlach, Die Intervention, Hamburg 1967, S. 29 ff.

2 Text: International Legal Materials 1966, S. 374-376.

3 Vgl. dazu S. Kovalev, Suverenitet i internacional'nye ob'juzannosti socialističeskichstran, in: Pravda, vom 26. November 1968. 
Konvention über einen Interventionsmechanismus gegen alle Formen der Rassendiskriminierung zu ratifizieren. Nach dieser „International Convention on the Elimination of All Forms of Racial Discrimination "4 erhält jeder Unterzeichnerstaat das Recht, innenpolitische Maßnahmen eines anderen Unterzeichnerstaates, die seiner Meinung nach rassendiskriminierend sind, vor einen besonderen Ausschuß der Vereinten Nationen zu bringen, der dann eine Untersuchung des Falles durchführt. Die Konvention sieht allerdings noch keinen Mechanismus für ein Eingreifen vor. Der Abschluß eines Verfahrens ist für sie ein Bericht an die Vollversammlung der Vereinten Nationen. Es scheint aber nicht abwegig zu erwarten, daß die Vollversammlung ihrerseits im Einzelfall den Staaten Zwangsmaßnahmen empfehlen wird.

\section{Die Theorie der kontinentalen Jurisdiktion}

Vor zwei Jahren veröffentlichte der ostafrikanische Völkerrechtler Ali A. Mazrui unter dem Titel „Towards a Pax Africana“ eine Studie über Ideologie und Ehrgeiz der afrikanischen Politik ${ }^{5}$. Mazrui geht davon aus, daß ähnlich wie in Indien auch in Afrika die Pax Britannica oder die Pax Europea einen Friedenszustand zwischen den alten Reichen, Fürsten und Stämmen dadurch herbeigeführt hätte, daß das Tragen von Waffen und die legitime Gewaltanwendung bei den europäischen Kolonialmächten und ihren Hilfsorganen monopolisert worden sei. Schon das hätte eine Herausforderung an die Afrikaner dargestellt, für die die Waffe das Symbol der Mannhaftigkeit und Freiheit gewesen sei. Es sei in großem Maße dem Vorbild Gandhis zu danken, daß die Afrikaner diese Herausforderung nicht mit Waffengewalt sondern mit gewaltlosem Widerstand erwidert hätten. Es sei jedoch falsch, daraus zu folgern, daß nach dem Rückzug der europäischen Kolonialmächte aus Afrika nun auch der gewaltlose Widerstand in Afrika zum letzten Mittel der Auseinandersetzung in den auswärtigen Beziehungen geworden ist. Selbst Indien habe trotz aller Beschwörungen Gandhis aufgerüstet, obwohl es immer noch die Friedfertigkeit der Gewaltanwendung vorziehe und alle Staaten der Welt zum Verzicht auf militärische Gewaltanwendung zu überreden versuche. Der heutige Präsident von Tansania, Julius Nyerere, hatte noch im Jahre 1961 erklärt, es sei falsch, daß einzelne afrikanische Staaten bewaffnete Streitkräfte aufbauten. Bei den gegebenen Kräfteverhältnissen könnten solche Streitkräfte niemals als sinnvoller Schutz gegen Angriffe von Kolonialmächten gerechtfertigt werden, "realistically, it can only be armed against another African state". Afrikanische Staaten bedürften lediglich einiger Polizeieinheiten für die innere Sicherheit, „as far as larger commitments are concerned, these should be on an African basis". Dennoch baute Tanganjika eine Armee auf. Aber dieser Widerspruch zwischen Reden und Handeln Nyereres ist im Zusammenhang mit der Frage nach dem Recht zur interafrikanischen Intervention nicht von Bedeutung. Interessant ist hier vielmehr die Behauptung Mazruis, diese Äußerungen Nyereres implizierten die Idee der „Pax Africana“, die Idee der militärischen Gewaltanwendung in Afrika unter einer kontinentalen gesamtafrikanischen Jurisdiktion. Die „Pax Africana“ ersetze sowohl die „Pax Europea“ der Kolonialzeit, als auch die „Pax Humana“ in Gestalt eines Gewaltmonopols der Vereinten Nationen, wie es im Kongo versucht worden sei.

4 Text: International Legal Materials 1966, S. 352-366

5 Ali A. Mazrui, Towards a Pax Africana - A Study of Ideology and Ambition, Chicago und London 1967. 
Die „Pax Africana“ ziele nicht nur auf die Verhältnisse zwischen den Staaten, sondern auch in den Staaten und in den restlichen Kolonien. Sie sei auf die Erhaltung und Durchsetzung der „rassischen Souveränität“ der Afrikaner gerichtet (S. 195 ff.).

Mazrui befaßt sich nicht nur mit der Notwendigkeit der „Pax Africana“, sondern auch mit ihrer juristischen Grundlegung. Er führt dazu aus:

"Nonetheless, it should not be inferred that the principle of racial sovereignty is intended to replace that of state sovereignty. Like the Monroe Doctrine, the principle of racial sovereignty merely introduces an additional dimension to the doctrine of non-intervention. To take a non-African example, Saudi and Egyptian 'intervention' in the Yemen was thus a 'domestic' affair of the Arabs according to racial sovereignty, but an external violation of the rights of the Yemenis according to state sovereignty. A new level of externality would have been introduced by any non-Arab intervention.

By the same token, any Ugandan 'interference' in Congolese affairs in 1964-65 was interstate interference, but American and Belgian 'interference' in the Congo was both interstate and interracial.

In conclusion, let us clarify further the relationship between this principle of racial sovereignty and the five concepts of self-government ... To the extent that colonial rule in Africa's experience has denoted the rule of one race over another, colonialism is, ... a violation of racial sovereignty. But what if Algeria's colonial status had been ended not by independence but by honest democratic integration with metropolitan France? Perhaps with more feeling than logic Nkrumah for one could not conceive of such a merger between different peoples.... For the moment suffice it to quote Nkrumah's emphatic if confused assertion that 'Algeria is African and will always remain so, in the same manner that France is French.' " (S. 38 f.)

Diese Ausführungen drängen die Frage auf, warum denn ein Algerier ein Afrikaner, und Algerien daher Objekt afrikanischer Intervention, ein Franzose aber nur unter anderem Europäer und daher nicht Objekt europäischer Intervention sein soll. Mazrui beantwortet diese Frage mit dem Hinweis auf die kommende gesamtafrikanische Föderation. Aus der panafrikanischen Vision leitet er die Rechtfertigung einer schon heute wirksamen kontinentalen Jurisdiktion ab. Bleibe es dahingestellt, ob ein Staat in statu nascendi schon gewisse Rechte auf seinem Gebiet hat ${ }^{6}$, so ist es aber immer noch zweifelhaft, ob denn wirklich die afrikanischen Staaten willens sind, heute eine solche gesamtafrikanische Föderation zu errichten. Selbst wenn man das unterstellt, ergeben sich gerade im Falle Algeriens die weiteren Fragen daraus, daß Algerien der Arabischen Liga angehört und sich als Teil der arabischen Nation empfindet, so daß es für zwei Parteien Interventionsobjekt sein könnte. Das gilt um so mehr als auch in arabischen Kreisen schon Ideen einer rassischen Souveränität entwickelt worden sind ${ }^{7}$, die vielleicht noch eher Berechtigung haben. Mazrui wendet sich daher auch besonders der Frage des Afrikanertums der Araber zu. Er legt dar, daß die meisten Araber in Afrika lebten und sich hier unauflöslich mit den negroiden Einwohnern des Kontinents vermischt hätten (S. 113 ff.).

$6 \mathrm{Vgl}$. dazu Jochen Abraham Frowein, Das De-facto-Regime im Völkerrecht - eine Untersuchung zur Rechtsstellung "nichtanerkannter Staaten“ und ähnlicher Gebilde, Köln und Berlin 1968, S. $188 \mathrm{ff}$.

7 Der Irak hat beispielsweise im Jahre 1963 den „progressiven “ arabischen Staaten vorgeschlagen, man sollte sich gegenseitig ermächtigen, im Falle einer von außen gesteuerten Revolution in einem der Staaten, in diesen einzumarschieren und die alte Regierung wieder an die Macht zu bringen. Vgl. Keesing's Contemporary Archives $19387 \mathrm{~A}$. Da die Steuerung einer Revolution von außen ein sehr elastischer Anknüpfungspunkt ist, böte eine solche Formel die Handhabe $\mathrm{zu}$ "progressiv“-arabischen Interventionen gegen jede autochthone traditionalistische Bewegung in den einmal revolutionierten arabischen Staaten. 
Wenn von dem Selbstbestimmungsrecht der Rasse gesprochen werde, so sei dies als Selbstbestimmungsrecht der Hautfarbe zu verstehen (S. 109). Natürlich gäbe es in Afrika starke Unterschiede der Hautfarbe, diese würden jedoch in der allen gemeinsamen Ablehnung der Herrschaft des „weißen Mannes“, des Europäers, aufgehoben, so daß die Rasse der nichtweißen Einwohner Afrikas gegen Europa entstehe. Diese Antithetik zu den Europäern begründe die Einheit Afrikas (S. 116 ff.). Die so definierten Afrikaner wollten ihre Probleme allein lösen, sie forderten die Anerkennung des Grundsatzes der kontinentalen Jurisdiktion. Der Kreis der Träger dieser kontinentalen Jurisdiktion werde durch die Mitgliedschaft in der Organisation für Afrikanische Einheit umschrieben (S. 118).

Die Doktrin der rassischen Selbstbestimmung und kontinentalen Jurisdiktion läßt deutlich eine Verwandtschaft zur amerikanischen Monroe-Doktrin erkennen, auf die auch Mazrui hinweist. Mazrui zieht auch sogleich Parallelen zwischen der Beteiligung Algeriens, des Sudans und der Vereinigten Arabischen Republik an den Kämpfen im Kongo im Jahre 1964 und der amerikanischen Intervention in der Dominikanischen Republik von 1965. Er meint allerdings, die Gemeinsamkeit zwischen Nord- und Lateinamerika bestehe in den gemeinsamen Namen. Die Monroe-Doktrin sei daher nicht kontinental sondern hemisphärisch konzipiert. Deshalb wäre es zwar ein Fall kontinentaler Jurisdiktion, wenn Kuba in der Dominikanischen Republik interveniert hätte. Die Intervention der Vereinigten Staaten von 1965 sei jedoch eine verbotene extrakontinentale Intervention gewesen, die beispielsweise einer chinesischen Intervention in Marokko zu vergleichen wäre (S. 124 f.). Gegen solche Argumentation sei die Frage erlaubt, ob denn nicht das Abstellen auf die Zugehörigkeit zu einem Kontinent an Zufälligkeiten geographischer Termini anknüpft, die ohne wesentliche politische Bedeutung sind und nur den Sinn von technischen Hilfsmitteln der geographischen Orientierung haben. Die Aufteilung der euro-afro-asiatischen Landmasse in drei Kontinente ist politisch völlig bedeutungslos, und es fragt sich dann, warum ein Zyprer oder Sizilianer Europäer, ein Tunesier oder Marokkaner aber Afrikaner und ein Libanese Asiate sein soll. Zur Antwort wird auf die den Kontinent einende Idee des Afrikanertums, d. h. die gemeinsame Gegnerschaft zu den "Weißen“ verwiesen. Mit gleichem Recht könnte man dann aber auch sofort eine afro-asiatische rassische Einheit postulieren. Es scheint, daß hier höchst problematische Formeln, genauer Antithesen, eines in seiner Fragwürdigkeit sonst auch von Afrikanern erkannten europäischen Weltbildes zum Fundament einer neuen völkerrechtlichen Doktrin gemacht werden sollen. Mit der vagen Idee des Afrikanertums wird ein Raum zur rechtlich relevanten Einheit erhoben. Selbst die Diktion Mazruis zeigt hier einige Unsicherheit, und die Studie beschränkt sich dann oft auf die Wiedergabe von Reden afrikanischer Politiker.

Trotz all dieser $Z$ weifel hat man zur Kenntnis zu nehmen, daß es sich hier um die erste geschlossene Formulierung einer afrikanischen Völkerrechtslehre handelt. Mazrui will auch mit seiner Studie die Grundlagen für ein afrikanisches Völkerrecht schaffen (S. 118 f.). Er geht davon aus, daß es für die auswärtigen Beziehungen drei Identitätskreise gibt, als obersten den rassischen, danach den kontinentalen und schließlich den des einzelnen Staates (S. 125). Jeder Kreis hat seine Souveränität, so daß Mazrui beispielsweise von kontinentaler Souveränität sprechen kann ( . 115). Mazrui betrachtet diese Kreise als einander überlagernde Jurisdiktionsbereiche. Der weiteste Jurisdiktionsbereich soll der rassische sein, der in Afrika mit dem kontinentalen Jurisdiktionsbereich zusammenfällt. Der engere Jurisdiktionsbereich besteht aus den einzelnen staatlichen Jurisdiktionsbereichen. Die verschiedenen Jurisdiktionsbereiche bilden eine einheitliche Ordnung, sie sind stufenweise Aus- 
bildungen eines rassischen Rechtssystems, in dem der höhere Bereich stets dem niederen übergeordnet ist. Die Theorie läuft also auf ein Primat der rassischen und kontinentalen Jurisdiktion gegenüber den einzelnen Staaten hinaus, so daß den einzelnen Staaten die rassische oder regionale Rechtsordnung vorgeht.

Aus dieser Konstruktion einer afrikanischen Regionalordnung zieht Mazrui bemerkenswerte Konsequenzen für das Recht zur interafrikanischen Intervention. Er behauptet, das traditionelle Völkerrecht sei nicht universell, sondern paneuropäisch konzipiert worden, es sei rassische oder kontinentale Jurisdiktion. Beispielsweise sei die Anerkennung einer Regierung nach dem klassischen Völkerrecht stets unter dem Gesichtspunkt erfolgt, ob die anzuerkennende Regierung in Gestalt und Habitus der Norm europäischer Staaten entspreche. Die Legitimität von Regierungen in Afrika sei ebenfalls aufgrund afrikanischer Normen zu prüfen. Die afrikanischen Normen für die Anerkennung einer Regierung seien:

1. Das Vorhandensein effektiver Kontrolle über ein bestimmtes Land;

2. Das Afrikanertum der Repräsentanten;

3. Die legitime Erlangung der Macht;

4. Die legitime Ausübung der Macht (allerdings nicht unbedingt nach den Maßstäben westlicher Demokratien);

5. Die Unabhängigkeit von nicht-afrikanischen Mächten.

Wenn eine Regierung diese Voraussetzungen nicht erfülle, hätten die übrigen afrikanischen Staaten das Recht zur Intervention im Interesse der Sicherheit der Afrikaner und ihres Kontinents (S. $122 \mathrm{ff}$.). Solche Interventionen, wie beispielsweise die Beteiligung Algeriens, des Sudans und der Vereinigten Arabischen Republik am Bürgerkrieg im Kongo (Leopoldville) im Jahre 1964, verstießen nicht gegen den Grundsatz der Souveränität, sie seien vielmehr eine aus dem Grundsatz der rassischen Souveränität folgende Ergänzung zur Lehre vom Interventionsverbot (S. 125).

Der Gedanke einer völkerrechtlich relevanten Einheit von Idee und Raum, der hinter der These von der kontinentalen Jurisdiktion unter rassischer Souveränität steht, findet in der europäischen Völkerrechtsgeschichte eine bemerkenswerte Parallele in Carl Schmitts These vom „völkerrechtlichen Großraum mit Interventionsverbot für raumfremde Mächte" 8 . Schmitt geht wie Mazrui von der Monroe-Doktrin aus (S. 12 ff.) und qualifiziert den Raum durch die Verbindung mit einer Idee, die „ein bestimmtes Volk ... trägt und ... einen bestimmten Gegner im Auge hat", zum völkerrechtlichen Topos (S. 19). In solchen Räumen soll der Träger der Idee das Recht zur Intervention gegen schädliche Elemente haben, ohne dabei auf die Souveränität von Staaten Rücksicht nehmen zu müssen (S. 51). Schmitt ist dabei allerdings konsequenter als Mazrui; er lehnt prinzipiell den Begriff des Staates als „Zentralbegriff des Völkerrecht" ab und setzt den Begriff des „Volkes“ an seine Stelle (S. 40 ff.), wie im Modell Mazruis das "Afrikanertum" zum zentralen Topos wird, auch wenn Mazrui sich noch schwer tut, den Begriff des Staates ganz über Bord zu werfen. „Volk“ oder „Afrikanertum" sind jedoch, wie schon die Definitionsversuche bei Mazrui zeigen, sehr vage Begriffe. Sie führen zur Manipulierbarkeit des Völkerrechts im Interesse einer ideologisch begründeten politischen Dynamik und nehmen dem Völkerrecht damit die Ansätze, durch feste Rechtssätze gesicherte auswärtige Beziehungen zu ermöglichen. Der Wert der Sicherheit für eine gedeihliche Entwicklung der internationalen

8 Carl Schmitt, Völkerrechtliche Großraumordnung mit Interventionsverbot für raumfremde Mächte - Ein Beitrag zum Reichsbegriff im Völkerrecht, 3. Aufl., Berlin, Leipzig und Wien 1941. 
Ordnung, auf den die Grundsätze der Souveränität und Gleichberechtigung aller Staaten im Völkerrecht ausgerichtet sind, wird in einem dynamischen System zugunsten einer "epochalen" Idee vernachlässigt, wie das auch bei der BreschnewDoktrin ausgeprägt ist und wie es die weiter unten noch zu erwähnende lateinamerikanische Larreta-Doktrin als Variation der Monroe-Doktrin ebenfalls erkennen ließ. Es fragt sich allerdings, inwieweit die afrikanischen Staaten in der Praxis heute bereit sind, die Idee des „Afrikanertums“ gesicherten internationalen Beziehungen auf der Basis der Souveränität der Staaten vorzuziehen.

\section{Die Haltung der afrikanischen Staaten zum Recht zur Intervention}

\section{Die Charta der Organisation für Afrikanische Einheit und die Intervention in Kolonialgebiete}

Nach der Charta der Organisation für Afrikanische Einheit ${ }^{9}$ ist zwar die Intervention eines Staates in einem anderen Staat verboten, denn die Mitgliedstaaten verpflichten sich zur bedingungslosen Beachtung der folgenden Grundsätze:

"1. the sovereign equality of all Member States;

2. non-interference in the internal affairs of States;

3. respect for the sovereignty and territorial integrity of each State and for its inalienable right to independent existence;

...

5. unreserved condemnation, in all its forms, of political assassination as well as of subversive activities on the part of neighbouring States or any other State;

..." (Art. III und Art. VI).

Damit wird aber nur ein Recht zur Intervention zwischen den Staaten ausgeschlossen. Unberührt bleibt von diesen Grundsätzen eine Intervention unter Berufung auf den Grundsatz der rassischen Souveränität und die kontinentale Jurisdiktion. Die Charta selbst bietet bereits die Rechtsgrundlage für den Einsatz der Organisation zur Durchsetzung der rassischen Souveränität. Gemäß Art. II 1. d. hat die Organisation die Aufgabe "to eridicate all forms of colonialism from Africa“. Und die Staaten verpflichten sich bedingungslos der "total emancipation of the African territories, which are still dependent" (Art. III 6. und Art. VI). Bereits auf der konstituierenden Konferenz der OAU im Mai 1963 wurde eine Entschließung zur Dekolonisierung gefaßt, in der es u. a. heißt:

"The Summit Conference of Independent African States ...

Reaffirming that it is the duty of all African Independent States to support dependent peoples in Africa in their struggle for freedom and independence; ... have decided on the following measures:

...

11. Establishes a Co-ordinating Committee consisting of Algeria, Ethiopia, Guinea, Congo (Leopoldville), Nigeria, Senegal, Tanganyika, United Arab Republic and Uganda, with Headquarters in Dar-es-Salaam, Tanganyika, responsible for harmonizing the assistance from African States and for managing the Special Fund to be set for that purpose.

12. Establishes a Special Fund to be raised by voluntary contribution of Member States ...; requests the Co-ordinating Committee to propose the

9 Text: International Legal Materials 1963, S. $766 \mathrm{ff}$. 
necessary fund and the appointment among Member States to the Council of Ministers so as to supply the necessary practical and financial aid to the various African national liberation movements;

...

14. Decides to receive on the territories of independent African States nationalists from liberation movements in order to give them training in all sectors and afford young people all the assistance they need for their education and vocational training;

15. Decides further to promote, in each State, the transit of all material aid and the establishment of a body of volunteers in various fields, with a view to providing the various African national liberation movements with the assistance they need in the various sectors." (Text bei Mazrui, S. $230 \mathrm{ff}$.) Mit der noch im Jahre 1963 erfolgten Verwirklichung dieser Resolution der OAU ist ein afrikanischer Interventionsapparat errichtet worden ${ }^{10}$. Selbst wenn einzelne Staaten an der Arbeitsweise des Apparates unter Hinweis auf das Mißverhältnis zwischen Kosten und Erfolgen Kritik geübt haben"11, ist von keinem "afrikanischen" Staat jemals die Unzulässigkeit solcher Interventionen in afrikanische Gebiete, die völkerrechtlich unter der Souveränität anderer, d. h. europäischer Staaten stehen, geltend gemacht worden. Die angenommene kontinentale Jurisdiktion der OAU scheint diese Interventionen im Rahmen rassischer Souveränität hinreichend zu rechtfertigen, zumal sie sich nicht gegen afrikanische Staaten richten, sondern gerade gegen deren radikalstes Gegenstück, nämlich europäische Herrschaft auf afrikanischem Boden.

\section{Die Kommission für Vermittlung, Versöhnung und Schlichtung}

Ein Interventionsmechanismus, der gegen afrikanische Staaten gerichtet werden kann, ist im Jahre 1964 von der Organisation für Afrikanische Einheit geschaffen worden, nämlich die bereits in der Satzung der Organisation als Organ der kontinentalen Jurisdiktion vorgesehene Kommission für Vermittlung, Versöhnung und Schlichtung ${ }^{12}$, die für alle innerafrikanischen zwischenstaatlichen Streitigkeiten zuständig ist (Art. XIX der Satzung; Art. XII des Protokolls). Die Kommission besteht aus einundzwanzig von der Versammlung der Staatschefs auf fünf Jahre gewählten Mitgliedern mit „anerkannten fachlichen Qualitäten“. Sie wird von einem Präsidenten und zwei Vizepräsidenten, die hauptberuflich tätig sind, geleitet. Das Präsidium hat hauptsächlich mit den Streitparteien ein geeignetes Verfahren zur Streitbeilegung zu beraten und die Verbindlichkeit einer etwaigen Entscheidung zu vereinbaren. Ahnliche Schiedsorgane gibt es auch in Amerika. Die Besonderheit der afrikanischen Kommission liegt allerdings darin, daß nicht nur die Streitparteien vor der Kommission aktiv legitimiert sind, sondern auch der Ministerrat der OAU und die Versammlung der Staatschefs, die mit Mehrheitsentscheidung jeden Streit zwischen "afrikanischen“ Staaten vor die Kommission bringen können (Art. XIII des Protokolls). Jede interafrikanische Spannung wird damit a priori zu einer Angelegenheit von gesamtafrikanischem Interesse erklärt und kontinentaler Jurisdiktion unterworfen. Auch wenn die Verbindlichkeit einer Entscheidung von der Zustimmung der Parteien abhängig bleibt, ist in der Aktivlegitimation konti-

10 Vgl. Andrew W. Scott, The Revolution in Statecraft - Informal Penetration, 2. Aufl., Chicago und

11 Vgl. dazu Dieter Schröder, die Konferenzen der „Dritten Welt“, Hamburg 1968, S. 131 und 134.

12 Protokoll über die Kommission in: International Legal Materials 1964, S. $1116 \mathrm{ff}$. 
nentaler Organe der Ansatz für Interventionen in die Auswärtigen Angelegenheiten afrikanischer Staaten zu sehen, die allerdings nicht gegen die Satzung der OAU verstößt, da diese nur Einmischungen in innere Angelegenheiten von Staaten verbietet. Es läßt sich bisher allerdings nicht beurteilen, inwieweit die Kommission zur Lösung interafrikanischer Streitigkeiten beigetragen hat. Bei einigen Grenzstreitigkeiten ist sie zwar eingeschaltet worden und hat durch ihre Untersuchungen zur Versachlichung des Streites segensreich beigetragen, die Lösungen wurden jedoch stets durch die Vermittlung dritter Staaten herbeigeführt. Die Kommission hat damit als Instrument kontinentaler Jurisdiktion und Intervention praktisch keine Bedeutung erlangt. Die Problematik der interafrikanischen Intervention liegt vielmehr in der Inanspruchnahme einer kontinentalen Jurisdiktion über die inneren Verhältnisse eines afrikanischen Staates.

\section{Die Intervention in innere Angelegenheiten „afrikanischer“ Staaten}

Für die kontinentale Jurisdiktion über die inneren Verhältnisse eines afrikanischen Staates hat die Organisation für Afrikanische Einheit keinen dem Befreiungsausschuß oder der Schiedskommission vergleichbaren Interventionsmechanismus entwickelt. Es läßt sich allerdings für diesen Bereich eine bemerkenswerte afrikanische Praxis erkennen. Die Fälle von interafrikanischen Interventionen in innere Angelegenheiten anderer afrikanischer Staaten erfolgten bisher stets außerhalb der OAU. Intervenienten waren Ghana, Kongo (Brazzaville) und andere vor allem progressive Staaten, die behaupteten, "neokolonialistische" Regierungen in anderen afrikanischen Staaten bedrohten die Sicherheit aller Afrikaner, daher seien alle afrikanischen Staaten verpflichtet, Freiheitskämpfer aus und in solchen Staaten zu unterstützen. In der Terminologie Mazruis handelte es sich um illegitime Regierungen, die nicht anerkennungs- und respektwürdig sind. Spektakulär wurde diese "wilde" interafrikanische Intervention mit dem Eingreifen Algeriens, des Sudans und der Vereinigten Arabischen Republik in die Kämpfe im Kongo (Leopoldville) im Jahre 1964 zu Gunsten der Gegner Tschombés. Die OAU protestierte zwar gegen europäische Einmischungen im Kongo, war aber nicht bereit, selbst im Kongo $\mathrm{zu}$ intervenieren. Der Schritt zu massiver interafrikanischer Intervention im Kongo erfolgte auf der zweiten Konferenz bündnisfreier Staaten, die im Jahre 1964 in Kairo stattfand. Die Vereinigte Arabische Republik, Jugoslawien und Ceylon forderten den kongolesischen Staatspräsidenten auf, das Erscheinen des kongolesischen Ministerpräsidenten und Außenministers Tschombé in Kairo zu verhindern. Sie verbanden die Aufforderung allerdings mit der Erklärung, sie sei „keineswegs als Versuch zur Einmischung in die inneren Angelegenheiten des Kongo zu verstehen"13. Der Kongo ignorierte die Aufforderung. Tschombé erschien als Delegationschef des Kongo (Leopoldville) in Kairo und wurde sofort von den Behörden der Vereinigten Arabischen Republik interniert. Daraufhin inhaftierte der Kongo seinerseits Diplomaten der VAR und Algeriens. Die Mehrheit der in Kairo versammelten afrikanischen Staatschefs ergriff die Partei der Intervenienten und forderte Kasavubu auf, Tschombé unverzüglich abzuberufen. Es wirkt grotesk, daß dieselbe Konferenz die „völlige Hingabe an das Grundprinzip der internationalen Beziehungen, demzufolge die Souveränität ... aller Staaten, ..., unantastbar ... ist" ${ }^{14}$ proklamierte.

13 Second Conference of Non-aligned Countries, in: Review of International Affairs 1964, No. 350, S. 5 f. 14 Programm für Frieden und internationale Zusammenarbeit, Belgrad 1964, S. 19. 
Im Jahre 1965 erreichte die Interventionspolitik Ghanas unter Nkrumah ihren Höhepunkt. Von Ghana aus wurden Partisanenverbände in der Elfenbeinküste, Kamerun, Niger, Obervolta und Togo zum Einsatz gebracht. Auf den Präsidenten von Niger, Hamani Diori, wurde, vermutlich mit ghanaischer Unterstützung, ein Mordanschlag versucht ${ }^{15}$. Gleichzeitig rückten die von den ghanaischen Interventionen hauptsächlich betroffenen frankophonen Staaten Afrikas enger zusammen und gründeten eine Afrikanisch-Madagassische Gemeinschaftsorganisation (OCAM). Bereits im Mai 1965 beschloß eine Sonderkonferenz dieser Organisation, den Kongo (Leopoldville) unter der Regierung Tschombés in die OCAM aufzunehmen. Sie erklärte gleichzeitig unaufgefordert ihre Bereitschaft, dem Kongo (Leopoldville) zu helfen, und rief alle anderen OAU-Mitgliedstaaten auf, die Aufständischen im Kongo nicht zu unterstützen ${ }^{16}$. Die OCAM erklärte damit faktisch, daß interafrikanische Interventionen nur aufgrund eines Ersuchens der amtierenden Regierung zulässig seien; sie wandte sich also gegen die Begründung einer Intervention aus einer Illegitimität einer amtierenden Regierung aufgrund „kolonialistischer" oder "neokolonialistischer" Verdächtigungen.

Die dritte Konferenz der Staatschefs der OAU sollte in demselben Jahr in Accra, der Hauptstadt Ghanas, stattfinden. Unter Hinweis auf die ghanaische Interventionspolitik lehnten jedoch die Elfenbeinküste, Dahome, Gabun, die Malagesische Republik, Niger, Obervolta, Togo und Tschad die Teilnahme an einer Konferenz in Accra ab, es sei denn, daß Ghana seine Interventionspolitik unverzüglich aufgäbe. Weitere Staaten entsandten demonstrativ kleine Gruppen von nachgeordneten Ministern oder Diplomaten (Burundi, Kenia, Libyen, Marokko, Ruanda, der Senegal, Tunesien, Uganda und die Zentralafrikanische Republik). Ordentlich vertreten waren auf der Konferenz vor allem die progressiven Staaten, die ehemals die Casablanca-Gruppe gebildet hatten (Algerien, Ghana, Guinea, Mali, der Sudan und die Vereinigte Arabische Republik) sowie Äthiopien, Gambia, Kamerun, Kongo (Brazzaville), Kongo (Leopoldville), Liberia, Malawi, Mauretanien, Nigeria, Sierra Leone, Somalia, Tansania und Sambia ${ }^{17}$. Die Konferenz von Accra war primär eine Versammlung anglophoner Staaten, was die Gefahr heraufbeschwor, daß in der Zukunft in Gestalt der frankophonen OCAM und einer anglophonen OAU zwei afrikanische Kontinentalorganisationen miteinander konkurrieren und nicht-afrikanischen Mächten Ansatzpunkte für verstärkte Einwirkungen in Afrika bieten würden. Auf der vierten Konferenz der OAU im Jahre 1966 verstärkte sich diese Entwicklung. Jetzt waren nur noch Äthiopien, Algerien, Gambia, Ghana, Kamerun, Lesotho, Liberia, Mali, Mauretanien, Sierra Leone, Sambia, Somalia, der Sudan, Tansania, die Vereinigte Arabische Republik und die Zentralafrikanische Republik durch ihre Staatschefs vertreten. Tunesien übermittelte der Konferenz den Vorschlag, das Co-ordinating Committee für die Befreiungsbewegungen aufzulösen, und die Konferenz beschloß, das Budget des Committees zu kürzen. Nyerere, der Präsident von Tansania, erklärte daraufhin nach der Konferenz, es habe sich gezeigt, daß Großbritannien und Frankreich in der OAU mehr Macht hätten, als ganz Afrika ${ }^{18}$. Auf der elften Sitzung des Committees wurden „certain OAU members" angeklagt, "who, instead of helping and encouraging their brother in the struggle, fraternize with the enemy" 19.

15 Vgl. Keesing's Contemporary Archives 20894 f.

16 Vgl. Keesing's Contemporary Archives 20893.

$17 \mathrm{Vgl}$. Keesing's Contemporary Archives 21051.

$18 \mathrm{Vgl}$. Keesing's Contemporary Archives 21738 B.

19 Vgl. Africa Research Bulletin 834 A. 
Die fünfte Konferenz der OAU, die im Jahre 1967 in Kinshasa (Kongo) zusammentrat, wurde fast vollständig von der OCAM beherrscht. Der nigerische Präsident Diori konnte daher als Sprecher der OCAM die Veranstaltung als „Konferenz des Realismus" und "Neubeginn der OAU“ bezeichnen" ${ }^{20}$. Er erklärte in einer programmatischen Rede, der zukünftige Erfolg der OAU hänge davon ab, daß vier Grundsätze beachtet würden, „die Nichteinmischung in die Angelegenheiten anderer Länder, die Achtung der nationalen Souveränität und Integrität, die friedliche Beilegung von Streitigkeiten, die Absage an politische Attentate und Subversion", nur so könne die OAU die innere Befriedung Afrikas herbeiführen². Damit sollte die Ablehnung der interafrikanischen Intervention zum Prinzip der OAU erhoben werden.

Den Prüfstein für die Beständigkeit dieser Entwicklung bildete die Nigeria-Krise des Jahres 1968. Die fünfte Konferenz der OAU hatte Sezessionen in afrikanischen Staaten verurteilt und anerkannt, daß solche Sezessionen innere Angelegenheiten des betroffenen Staates seien 22. Das war jedoch nicht die einhellige Auffassung der OAU. Im Frühjahr 1968 anerkannte vielmehr Tansania, einer der Staaten, die der fünften Konferenz der OAU ferngeblieben waren, die Ostregion Nigerias als die „independent sovereign entity“ Biafra. Die tansanische Regierung fügte erläuternd hinzu, das Volk von Biafra habe sich zur Eigenexistenz entschlossen. Kein Staat sei berechtigt, ihm im Namen der afrikanischen Einheit sein Recht zu verweigern ${ }^{23}$. Die nigerianische Zentralregierung erklärte dieses Vorgehen als aggressiven Akt. Mali und Obervolta schlossen sich dem an ${ }^{24}$. Alsbald folgten Anerkennungen Biafras durch Gabun, die Elfenbeinküste und Sambia, die alle mit der Hoffnung ausgesprochen wurden, so dem Bürgerkrieg ein schnelles Ende zu bereiten, und mit der Begründung, daß auf dem Gebiet von Biafra nun schon über Monate eine eigene Regierung - gestützt auf den Consens der Bevölkerung - herrsche, daß also ein Staat entstanden sei25. Die Biafra anerkennenden Staaten haben sich jedoch nicht zur Modifizierung der Ablehnung von Interventionen entschlossen, sondern lediglich festgestellt, daß ihrer Meinung nach der nigerianische Bürgerkrieg zum Krieg zwischen zwei Staaten geworden sei. Schon in der tansanischen Erklärung hatte es geheißen, daß solche Entwicklungen in der Geschichte nicht ungewöhnlich seien und von einem Staat, der das Selbstbestimmungsrecht der Völker anerkenne, nicht im Namen kontinentaler Einheit behindert werden dürften. Auch dies war also eine Absage an das Prinzip des Selbstbestimmungsrechts der Rasse und die aus ihm abgeleitete kontinentale Jurisdiktion über die inneren Angelegenheiten von afrikanischen Staaten. Durch die Anerkennung Biafras war die Nigeria-Krise zu einer Auseinandersetzung zwischen zwei afrikanischen Staaten geworden, die jetzt als auswärtige Angelegenheit afrikanischer Staaten unter die Jurisdiktion der OAU fällt. Auf der nächsten Konferenz der OAU im Herbst 1968 in Algier forderte dann auch Tunesien, das der OCAM nahesteht, eine Einschaltung der OAU in die Nigeria-Krise, während die Mehrheit sich einem solchen Vorgehen widersetzte ${ }^{26}$. Gabun hat zwar seither das von ihm als Staat anerkannte Biafra erheblich materiell unterstützt, aber die große Mehrheit der afrikanischen Staaten hält sich nach wie vor zurück.

\footnotetext{
20 Nach Dieter Döllken, Die OAU-Konferenz in Kinshasa, in: Afrika heute 1967, S. $277 \mathrm{f}$.

21 Nach „Perspectives nigériennes ${ }^{*}$, Paris, Oktober 1967, S. 2.

$22 \mathrm{Vgl}$. Keesing's Contemporary Archives 22281 A.

$23 \mathrm{Vgl}$. Africa Research Bulletin $1040 \mathrm{~A}$.

24 Vgl. Keesing's Contemporary Archives 22672 A

25 Vgl. Keesing's Contemporary Archives 22880.

$26 \mathrm{Vgl}$. Africa Research Bulletin $1173 \mathrm{ff}$.
} 
Die Haltung der afrikanischen Staaten in der Nigeria-Krise zeigt, daß sie mittlerweile die interafrikanische Intervention als problematisch erkannt haben und sogar bereit sind, unter Außerachtlassung des für die jüngste Geschichte Afrikas so bedeutsamen Selbstbestimmungsgrundsatzes ganz positivistisch die Existenz der vorhandenen Staaten zu sichern. In jedem dieser Staaten gibt es mehr oder weniger starke Bevölkerungsgruppen, die unzufrieden sind und zu Aufständen übergehen können. Die Anerkennung eines Rechts auf interafrikanische Intervention unter kontinentaler Jurisdiktion würde solche Gruppen ermuntern und damit eine Vielzahl von weiteren Krisenherden in Afrika provozieren, die wiederum auch außerafrikanischen Mächten, wie im Kongo und in Nigeria erkennbar wurde, Gelegenheit zur Einflußnahme in Afrika geben würden. Die Befriedung Afrikas und die unter dem Schlagwort „Bündnisfreiheit“ betriebene Politik der Reserve gegenüber den Großmächten wären gefährdet. Außerdem wäre jede afrikanische Regierung gezwungen, ihre innenpolitischen Maßnahmen an den Vorstellungen der OAU zu orientieren. Sie würde ihre Handlungsfreiheit, ihre Souveränität, verlieren. Eine „Status-quo"-Politik wird damit zur Generallinie der OAU.

Es ist zu vermuten, daß die Doktrin von der rassischen Souveränität und von der kontinentalen Jurisdiktion revidiert wird in Richtung auf ein striktes Interventionsverbot. Sicher dürfte jedenfalls jetzt schon sein, daß es kein allgemein anerkanntes Recht zur interafrikanischen Intervention als regionales Völkerrecht in Afrika gibt. Damit ist allerdings noch nicht gewiß, ob auch die Doktrin von der rassischen Souveränität als Rechtfertigung von Interventionen gegen Kolonien nicht-afrikanischer Mächte in Afrika aufgegeben wird. Immerhin haben im Jahre 1965 die afrikanischen Staaten einmütig beschlossen, in Großbritannien gegen die RhodesienPolitik zu intervenieren, aber nur neun von 36 Staaten den als Anfang vereinbarten Schritt getan und die diplomatischen Beziehungen abgebrochen ${ }^{27}$. In jüngster Zeit haben sogar Botswana, die Elfenbeinküste, Lesotho, die Madagassische Republik und Malawi zu Südafrika diplomatische Beziehungen aufgenommen bzw. Interesse an der Aufnahme solcher Beziehungen zu erkennen gegeben ${ }^{28}$. Bei Botswana und Lesotho ist eine solche Haltung noch aus der Lage erklärlich; die Elfenbeinküste, die Madagassische Republik und Malawi sind von solchen Rücksichten jedoch frei. Die führende Stellung der Elfenbeinküste in der OCAM läßt es als nicht ausgeschlossen erscheinen, daß die frankophonen Staaten auf längere Sicht auch eine Revision der Interventionspolitik gegen nicht-afrikanisch beherrschte Gebiete vollziehen werden. Die sechste Konferenz der OAU hat zwar wieder gegen Südafrika Stellung genommen, sie hat aber auch eingewilligt, daß ein Ausschuß aus Dahome, Ghana, Liberia und Sierra Leone die Arbeit des Co-ordinating Committee überprüft ${ }^{29}$. Dahome ist mit der Elfenbeinküste in der westafrikanischen Entente verbunden. Ghana ist um enge Beziehungen zur Elfenbeinküste bemüht ${ }^{30}$ und auch Liberia und Sierra Leone können nicht gerade als progressive Staaten gelten. Auch in der Kolonialfrage scheint eine Hinwendung der afrikanischen Staaten zur "Status-quo"-Politik nicht mehr ganz unmöglich.

27 Vgl. Keesing's Contemporary Archives 21181.

28 Vgl. Africa Research Bulletin - Political, Social and Cultural Series 695 C; 879 C; 1000 A f.

29 Vgl. Africa Research Bulletin - Political, Social and Cultural Series $1172 \mathrm{ff}$.

$30 \mathrm{Vgl}$. Africa Research Bulletin - Political, Social and Cultural Series $1176 \mathrm{~B} f$. 


\section{Zusammenfassung}

Die augenblickliche Haltung der afrikanischen Staaten zum Recht zur Intervention in Afrika läßt sich demnach wie folgt zusammenfassen:

Interventionen sind grundsätzlich unzulässig, abgesehen von kritischen Stellungnahmen kontinentaler Organe zu auswärtigen Konflikten zwischen kontinentalen Staaten. Diese Stellungnahmen dürfen jedoch nicht die Souveränität eines kontinentalen Staates einschränken.

Ungewiß ist die zukünftige Haltung zur Intervention kontinentaler Organe gegen die Herrschaft außerkontinentaler Mächte auf dem Kontinent.

Damit zeigt sich, daß die afrikanischen Staaten nach anfänglichem Zögern auf dem Wege sind, das Prinzip der Souveränität der Staaten im Sinne des klassischen Völkerrechts über die Grundsätze von rassischer Souveränität und kontinentaler Jurisdiktion zu stellen. Innerhalb eines Jahrzehnts sind die afrikanischen Staaten von einem aus dem antikolonialen Befreiungskampf resultierenden, naturrechtlich begründeten radikalen Interventionsdenken zu einem auf Bewahrung von Frieden und Sicherheit orientierten Positivismus gelangt. Diese Entwicklung zu staatsmännischer Einsicht verdient die größte Achtung, wenn man bedenkt, daß die USA mehr als ein Jahrhundert benötigten, um zu begreifen, daß ein maßvoller Positivismus eine unabdingbare Voraussetzung für die Bewahrung des Friedens ist ${ }^{31}$. Noch nach 1945 unterstützten die USA die der kontinentalen Jurisdiktion ähnliche Doktrin des uruguayischen Außenministers Larreta, die kollektive Intervention, vor allem seitens der OAS, mit der Durchsetzung der "amerikanischen Werte" und der Annahme der Gefährdung des Friedens in der westlichen Hemisphäre durch die Existenz „unamerikanischer" Regime begründen wollte.

Es scheint jedenfalls nicht ganz unmöglich, daß die Pax Africana statt auf die Macht einer von verschiedenen Gruppen umkämpften kontinentalen Organisation auf die Vernunft der afrikanischen Politiker begründet wird. Interafrikanische Konflikte werden damit nicht ausgeschlossen, ihre erträgliche Lösung durch rationalen Interessenausgleich wird jedoch möglich gemacht. Wieweit sich die Rationalisierung der auswärtigen Beziehungen zwischen den afrikanischen Staaten auch auf die Beziehungen zu nicht-afrikanischen Staaten auswirkt, ist noch ungewiß. Es ist durchaus möglich, daß gegenüber diesen Staaten, die im Bewußtsein der afrikanischen Politiker als Gegner im antikolonialen Befreiungskampf und als negativer Pol der nationalen Integration erscheinen, die naturrechtliche Doktrin der rassischen Souveränität noch über lange Zeit aufrechterhalten wird.

31 Vgl. Knud Krakau, Missionsbewußtsein und Völkerrechtsdoktrin, Frankfurt am Main und Berlin 1967, S. $146 \mathrm{ff}$; ; aber auch die kritischen Außerungen der lateinamerikanischen Staaten bei Camara Filho, A Douctrina Laretta, in: Boletino da Sociedad Brasiliana de Direjo Internacionale 1946, S. 55 ff.; Carrizosa, La Crisis del Derecho Americano, in: Foro Internacionale 1961/62, S. $423 \mathrm{ff}$. 